\title{
GEODESICS IN THE BRAID GROUP ON THREE STRANDS
}

\author{
LUCAS SABALKA ${ }^{1}$
}

\begin{abstract}
We study the geodesic growth series of the braid group on three strands, $B_{3}:=\langle a, b \mid a b a=b a b\rangle$. We show that the set of geodesics of $B_{3}$ with respect to the generating set $S:=\{a, b\}^{ \pm 1}$ is a regular language, and we provide an explicit computation of the geodesic growth series with respect to this set of generators. In the process, we give a necessary and sufficient condition for a freely reduced word $w \in S^{*}$ to be geodesic in $B_{3}$ with respect to $S$. Also, we show that the translation length with respect to $S$ of any element in $B_{3}$ is an integer.
\end{abstract}

\section{INTRODUCTION}

For a finitely generated group $G$ the Geodesic Growth Series of $G$ with respect to a generating set $S=S^{-1}$ is defined by

$$
\mathcal{G}_{(G, S)}(x)=\sum_{n=0}^{\infty} a_{n} x^{n},
$$

where $a_{n}$ is the number $d_{S}$-geodesic words of length $n$ in $S^{*}$ and where $d_{S}$ is the word metric on $G$ defined by $S$. Similarly, the Spherical Growth Series is defined by

$$
\mathcal{S}_{(G, S)}(x)=\sum_{n=0}^{\infty} b_{n} x^{n},
$$

where $b_{n}$ is defined to be the number of elements of $G$ at $d_{S}$-distance $n$ from 1. A power series is rational if it may be expressed as a quotient of two integer-coefficient polynomials in the ring of formal power series $\mathbb{Z}[[x]]$. It is well known that the growth series of a regular language is rational 9 .

Note that regularity of the set of geodesics depends on the generating set used (14, attributed to Cannon). It is also known that the rationality of the spherical growth series depends on the generating set used [17.

Which groups have rational geodesic growth series? There are many groups which are known to have rational spherical growth series. Groups which have rational geodesic growth series are less well understood, but there are still many examples. It is known, for instance, that all discrete

\footnotetext{
${ }^{1}$ This work was done under the supervision of Drs. Susan Hermiller and John Meakin at the University of Nebraska-Lincoln, with funding from National Science Foundation grant DMS-0071037 and from a Pepsi UCARE grant.
} 
co-compact hyperbolic groups [3] have rational geodesic growth series. More generally, Neumann and Shapiro proved all geometrically finite hyperbolic groups have rational geodesic growth series [14, and all word-hyperbolic groups have rational geodesic growth series, independent of generating set (see [9]; attributed there to Gromov and others). Also, Loeffler, Meier, and Worthington proved this fact for right-angled Artin and Coxeter groups [13, and Noskov showed that irreducible affine Coxeter groups have rational geodesic growth series [15. Note these references actually show the regularity of the set of geodesics, which implies rationality of the geodesic growth series.

In this paper, we analyze the geodesic growth series of the braid group on three strands, $B_{3}:=\langle a, b \mid a b a=b a b\rangle$ (for a detailed description of $B_{3}$, see 9]). We prove that $B_{3}$ has a rational geodesic growth series with respect to the generating set $S=\{a, b\}^{ \pm 1}$ by showing the geodesics are a regular language. Moreover, we provide an explicit computation of the geodesic growth series with respect to $S$.

In fact, it has been shown (independent of this work) that $B_{3}$ has a rational geodesic growth series, although the actual geodesic growth series has not been computed. This was done by REU students Griffing, Patlovany, and Talley under the guidance of Jon McCammond [11. Charney and Meier have also recently proven that the geodesic growth series is rational for all Garside groups (which includes the braid groups), but on different generating sets than those considered here [4].

We prove the following theorems:

Theorem 1.1. A freely reduced word $w \in S^{*}$ is a geodesic for $B_{3}$ if and only if $w$ does not contain as subwords any of the following:

- elements of both $\{a b, b a\}$ and $\{A B, B A\}$;

- both aba and either $A$ or $B$;

- both $A B A$ and either a or b.

Theorem 1.2. The set of geodesics for $B_{3}:=\langle a, b \mid a b a=b a b\rangle$ with respect to the generating set $S=\{a, b\}^{ \pm 1}$ is regular, and the geodesic growth series of $B_{3}$ is

$$
\mathcal{G}_{\left(B_{3}, S\right)}(x)=\frac{x^{4}+3 x^{3}+x+1}{\left(x^{2}+x-1\right)\left(x^{2}+2 x-1\right)} .
$$

It is not known whether the geodesic growth series is rational for all braid groups.

This paper is organized as follows. In $\S 2$, background definitions are given. In section $\S 3$ we introduce $B_{3}$ and construct its Cayley graph. In section $\S 4$ we provide an analysis of possible geodesics in $B_{3}$ to prove Theorem 1.1 and we prove the translation length with respect to $\{a, b,\}^{ \pm 1}$ of any element of $B_{3}$ is an integer. In section $\S 5$ we give the proof of Theorem 1.2.

I would particularly like to thank Susan Hermiller and John Meakin as well as Ilya Kapovich for all of their time, advice, and help. 


\section{BACKGROUND}

Let $G$ be a group with a generating set $S=S^{-1}$. For any word $w \in S^{*}$ we denote by $\bar{w}$ the element of $G$ represented by $w$. If $w, v \in S^{*}$ are two words such that $\bar{w}=\bar{v}$, we write $w \equiv v$. Given a nontrivial freely reduced word $w \in S^{*}, w$ may be written in the form $w=w_{1} w_{2} \ldots w_{m}$, where each $w_{j}$ is of the form $s^{k}$ for some $s \in S$ and $k \in \mathbb{Z}, k \neq 0$, and where for $1 \leq j<m$, if $w_{j}=s^{k}$ then $w_{j+1}=t^{l}$ for some $l \neq 0$ with $t \neq s$. We call $w_{j}$ the $j$ th syllable of $w$. For any $x \in S$, we often use capitalization, $X$, to denote $x^{-1}$. If $w$ is a word or a path, we denote the length of $w$ by $|w|$.

Throughout this paper, we will use the term graph to mean a directed edge-labelled graph. Denote the set of vertices of a graph $M$ by $V M$ and the set of edges by $E M$. Also, let an edge $e \in E M$ from $v \in V M$ to $w \in V M$ labelled by $s$ be denoted $e=[v, w, s]$.

The main tool we will use to determine the geodesic growth series for $B_{3}$ is its Cayley graph. The Cayley graph of a group $G$ with respect to a generating set $S=S^{-1}$ is denoted $C_{(G, S)}$. In this paper we will only work with the situation when $S$ is partitioned as $S=S_{0} \sqcup S_{0}^{-1}$. We will refer to elements of $S_{0}$ as positive labels. If an edge is labelled with an element of $S_{0}$, it is called positive (or negative otherwise). Similarly, if a syllable of a word is an element of $S_{0}$ raised to a positive power, or an element of $S_{0}^{-1}$ raised to a negative power, it is also called positive. If a syllable is raised to an even (respectively, odd, negative, or positive) power, we say the syllable is even (respectively, odd, negative, positive). When drawing or defining a Cayley graph we will normally just indicate the positively labelled edges. If a specific generating set is understood, we simply write $C_{G}$ for the Cayley graph of $G$. In a Cayley graph $C=C_{(G, S)}$, a vertex $v \in V C$ is represented by a word $w \in S^{*}$ if there is a path $p$ with label $w$ beginning at 1 and ending at $v$ in $C$. In this case, we do not distinguish between $p$ and its label $w$. For any vertex $v \in V C$ represented by $w^{\prime}$ in $S^{*}$ and any word $w \in S^{*}$, we denote by $v w$ the vertex in $V C$ represented by $w^{\prime} w$.

If $p$ is of minimal length among all paths with the same end points, then $p$ is called geodesic in $C_{G}$. Similarly, a word $w \in S^{*}$ which is of minimal length among all words $w^{\prime} \in S^{*}$ for which $w^{\prime} \equiv w$ is called geodesic in $G$ (with respect to $S$ ). For a given element $g \in G$, if the word $w \in S^{*}$ is such that $\bar{w}=g$ and $w$ is a geodesic in $G$, then the cone type of $g$, and of the vertex in the Cayley graph which represents $g$, is defined as the set of all strings $\gamma$ such that $w \gamma$ is a geodesic in $G$. It is not hard to see that the cone type of $g$ does not depend on the choice of the geodesic $w$.

The short-lex ordering < on the set of all words $S^{*}$ over a finite set $S$ is a total ordering defined as follows. For $v, w \in S^{*}, v<w$ if $|v|<|w|$ or if $|v|=|w|$ but $v$ comes before $w$ lexicographically using some given lexicographic order on $S$. Let $G$ be a group with finite generating set $S$. For every $g \in G$ the smallest word $w \in S^{*}$ representing $g$ (with respect to the short lex ordering on $S^{*}$ ) is called the short-lex normal form of $g$. Note that 
if the set of short-lex normal forms of $G$ is a regular language then $\mathcal{S}_{(G, S)}$ is rational.

Let $G$ be a group with a given generating set $S$. Let $L_{G} \subset S^{*}$ denote the set of geodesic words in $G$. If $L_{G}$ is a regular language then $\mathcal{G}_{(G, S)}$ is rational. Then we may construct a finite state automaton (FSA) accepting $L_{G}$, and from the FSA we may compute a closed form of $\mathcal{G}_{(G, S)}$ :

Proposition 2.1 (9]). Let $G$ be a group with respect to a finite generating set $S=S^{-1}$. Assume $L_{G}$ is a regular language. Let $H$ be an FSA which accepts $L_{G}$. Let $M$ be the $n \times n$ adjacency matrix of $H$ minus fail states, with the first row of $M$ representing the adjacencies of the start state. Then:

$$
\mathcal{G}_{(G, S)}(x)=\sum_{k=0}^{\infty} a_{k} x^{k}=v_{1}(I-M x)^{-1} v_{2},
$$

where $I$ is the $n \times n$ identity matrix, $v_{1}$ is the $1 \times n$ row vector with a one in the first column and zeros elsewhere, and $v_{2}$ is the $n \times 1$ column vector of all ones.

We use this theorem to compute $\mathcal{G}_{\left(B_{3}, S\right)}$ with $S=\{a, b\}^{ \pm 1}$. Thus we first characterize geodesics for $B_{3}$, and then compute the appropriate FSA.

\section{The Cayley Graph of $B_{3}$}

3.1. Background. Recall $B_{3}:=\langle a, b \mid a b a=b a b\rangle$. To construct the Cayley graph for $B_{3}$, we will need the following definitions and theorem.

Note that in $B_{3}$, we have that $(b a b) b \equiv(a b a) b=a(b a b) \equiv a(a b a)$, and that $(a b a) a \equiv(b a b) a=b(a b a) \equiv b(b a b)$. In words, we may 'move' any occurrence of $(a b a) \equiv(b a b)$ around in a word representing an element of $B_{3}$ by interchanging the generators $a$ and $b$. We give this property of $B_{3}$ a name:

Definition 3.1 (The Garside property). The Garside property of $B_{3}$ refers to the relations $(a b a) x^{n} \equiv y^{n}(a b a)$ and $(A B A) x^{n} \equiv y^{n}(A B A)$.

Definition 3.2 (Right-greedy canonical form). [9] Let $S=\{a, b,\}^{ \pm 1}$. A word in $w \in S^{*}$ is in right-greedy canonical form for $B_{3}$ if $w=w_{1} w_{2} \ldots w_{m}$ where for each $i, w_{i} \in\{a, b, a b, b a, a b a, A B A\}$, and for $i<m$ :

- If $w_{i} \in\{a, b a\}$, then $w_{i+1} \in\{a, a b, a b a, A B A\}$.

- If $w_{i} \in\{b, a b\}$, then $w_{i+1} \in\{b, b a, a b a, A B A\}$.

- If $w_{i}=a b a$, then $w_{i+1}=a b a$.

- If $w_{i}=A B A$, then $w_{i+1}=A B A$.

Denote by $R G$ the set of words in right-greedy canonical form.

Note that this definition is specifically for $B_{3}$; in 9 , a more general definition is given. A word $w \in R G$ is thus of the form

$$
w=a^{k_{1}} b^{k_{2}} \ldots a^{k_{n-1}} b^{k_{n}}(a b a)^{j},
$$


with $j \in \mathbb{Z}, k_{i}>1$ for $1<i<n$, and $k_{1}, k_{n} \geq 0$. Every $g \in B_{3}$ can be represented uniquely by a word in right-greedy canonical form [9].

Proposition 3.3 ([7, 6]). The subgroup $\left\langle a^{2}, b^{2}\right\rangle$ of $B_{3}$ is isomorphic to the free group on two elements.

This is Tits' conjecture for $B_{n}$ where $n=3$. For $B_{n}$ with $n \leq 5$, this result was proven in 7 . Tits' conjecture was proven in full generality in 6 . Note Proposition 3.3 also follows from the canonicity of the right-greedy form.

3.2. The Graph $\Delta$. Consider the quotient group $G_{3}$ of $B_{3}$ obtained by adding the relation $a^{2} b^{2}=b^{2} a^{2}$ :

$$
G_{3}=\left\langle a, b \mid a b a=b a b, a^{2} b^{2}=b^{2} a^{2}\right\rangle .
$$

Various results about this group $G_{3}$ are proven in [16], including a construction of the Cayley graph $\Gamma=C_{G_{3}}$ of $G_{3}$, where $\Gamma$ was built by 'sewing' together translates of the Cayley graph of the free abelian group on $\left\langle a^{2}, b^{2}\right\rangle$ in a certain way. The following construction of the Cayley graph $C_{B_{3}}$ of $B_{3}$ is motivated by the graph $\Gamma$, where the Cayley graph of the free abelian group on $\left\langle a^{2}, b^{2}\right\rangle$ is replaced by the Cayley graph of the free group on $\left\langle a^{2}, b^{2}\right\rangle$ (see Figure 1). The reader may find it enlightening to keep $\Gamma$ in mind when reading the following description, as $\Gamma$ may be embedded in $\mathbb{R}^{3}$, and is thus easier to picture.

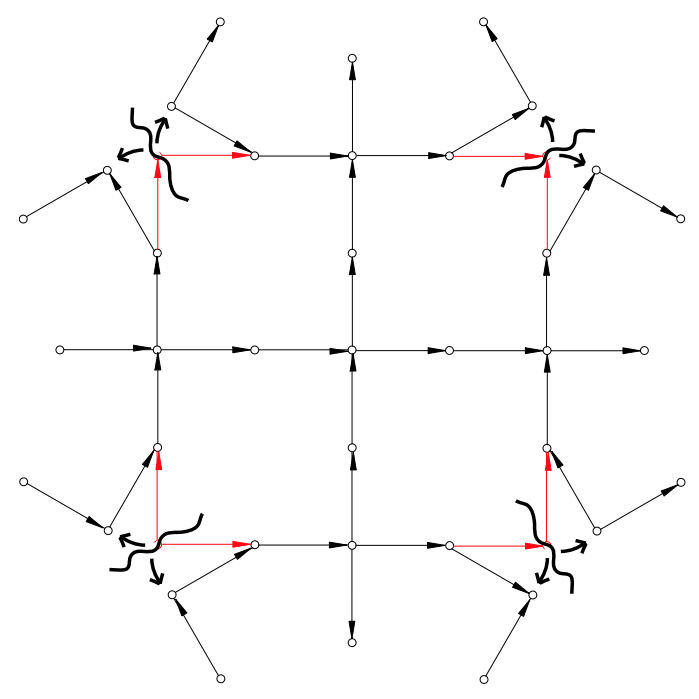

Figure 1. Obtaining $C_{B_{3}}$ from $C_{G_{3}}$.

Definition 3.4 (The graph $T^{\prime}$ ). Let $T$ be the Cayley graph $C_{F_{2}}$ of the free group on two generators $a$ and $b$. Let the graph $T^{\prime}$ be defined to be the subtree of $T$ spanned by $\left\langle a^{2}, b^{2}\right\rangle$. 
To form the Cayley graph for $B_{3}$, we want to take a countable number of translates of $T^{\prime}$ (one for each $k \in \mathbb{Z}$ ) and 'sew' them together. To do this, we define a graph $\Delta$ to have vertex set $\bigsqcup_{k \in \mathbb{Z}}\left(V T^{\prime}\right) \times\{k\}$. For every $k \in \mathbb{Z}$, let $\left(E T^{\prime}\right) \times\{k\}$ denote the set of edges induced by the inclusion $V T^{\prime} \rightarrow\left(V T^{\prime}\right) \times\{k\}$.

A vertex $v \in T^{\prime}$ is called an a-vertex (respectively, b-vertex) if $v$ has exactly two incident positive edges in $T^{\prime}$, both labelled by a (respectively, $b)$. Define the map $\lambda: S^{*} \rightarrow S^{*}$ to be the map induced by $\lambda(a)=b, \lambda(b)=a$, $\lambda(A)=B, \lambda(B)=A$. Then $\lambda^{2}(w)=w$. Consider any $v \in V T^{\prime}$ with $w$ a path from 1 to $v$ in $T^{\prime}$ for some $w \in S^{*}$. If $v$ is an $a$-vertex (respectively, a $b$-vertex), then let $\tilde{v}$ be the vertex represented by $\lambda(w) B A$ (respectively, $\lambda(w) A B)$ in $T^{\prime}$.

Definition 3.5 (The graph $\Delta$ ). Define the graph $\Delta$ as follows:

- $V \Delta:=\bigsqcup_{k \in \mathbb{Z}}\left(V T^{\prime}\right) \times\{k\}$

- The set of positively labelled edges of $\Delta$ is $\left(\bigcup_{k \in \mathbb{Z}}\left(E T^{\prime}\right) \times\{k\}\right) \cup$

$$
\begin{aligned}
& \left(\bigcup_{(v, k) \in V \Delta}[(v, k),(\tilde{v}, k+1), a], \mid v \text { is a } b \text {-vertex }\right) \cup \\
& \left(\bigcup_{(v, k) \in V \Delta}[(v, k),(\tilde{v}, k+1), b], \mid v \text { is an } a \text {-vertex }\right)
\end{aligned}
$$

(see Figure 2).

It is perhaps worth noting that this construction is related to the fact that the pure braid group $P_{3}$ is isomorphic to $F_{2} \times \mathbb{Z}$, where the $F_{2}$ subgroup of $P_{3}$ is exactly $\left\langle a^{2}, b^{2}\right\rangle$.

Let a word $w \in S^{*}$ be called almost even if every syllable of $w$ is even except possibly the last syllable.

Proposition 3.6. For every vertex $\left(v_{0}, k\right) \in V \Delta$, we may associate to $\left(v_{0}, k\right)$ a unique freely reduced word $w \in S^{*}$ from 1 to $(u, 0)$ and whence to $\left(v_{0}, k\right)$ in $\Delta$ of the form $w=w^{\prime}(a b a)^{k}$, where $w^{\prime}$ is almost even and $k \in \mathbb{Z}$.

Proof. Consider a vertex $\left(v_{0}, k\right) \in V \Delta$. Let $w^{\prime} \in S^{*}$ be a freely reduced word such that $\overline{w^{\prime}}=v_{0}$ in $T^{\prime}\left(w^{\prime}\right.$ is unique since $T^{\prime}$ is a tree). Note that in $\Delta$, the path labelled $a b a$ beginning at $\left(v_{0}, k\right)$ ends at $\left(v_{0}^{\prime}, k+1\right)$, where $v_{0}^{\prime}$ is the vertex represented by $\lambda\left(w^{\prime}\right)$ in $T^{\prime}$. Let $u$ be the vertex in $V T^{\prime}$ represented by $\lambda^{k}\left(w^{\prime}\right)$ (i.e. $u=v_{0}$ if $k$ is even, and $u=v_{0}^{\prime}$ if $k$ is odd). Consider the path $w:=\lambda^{k}\left(w^{\prime}\right)(a b a)^{k}$ in $\Delta$. The path $w$ is a path from $(1,0)$ to $(u, 0)$ and whence to $\left(v_{0}, k\right)$, where the first part of $w-\lambda^{k}\left(w^{\prime}\right)$ - is also a path in $T^{\prime}$. In $\Delta$, this means $\lambda^{k}\left(w^{\prime}\right)$ only connects vertices in $\Delta$ of the form $(v, 0)$. To stay in $T^{\prime}, \lambda^{k}\left(w^{\prime}\right)$ must be almost even, by the definition of $T^{\prime}$.

Let $C F$ (for 'Cartesian Form') denote the set of words $w$ with $w=$ $w^{\prime}(a b a)^{k}$ where $w^{\prime}$ has only even syllables except possibly the last syllable, and $k \in \mathbb{Z}$. 


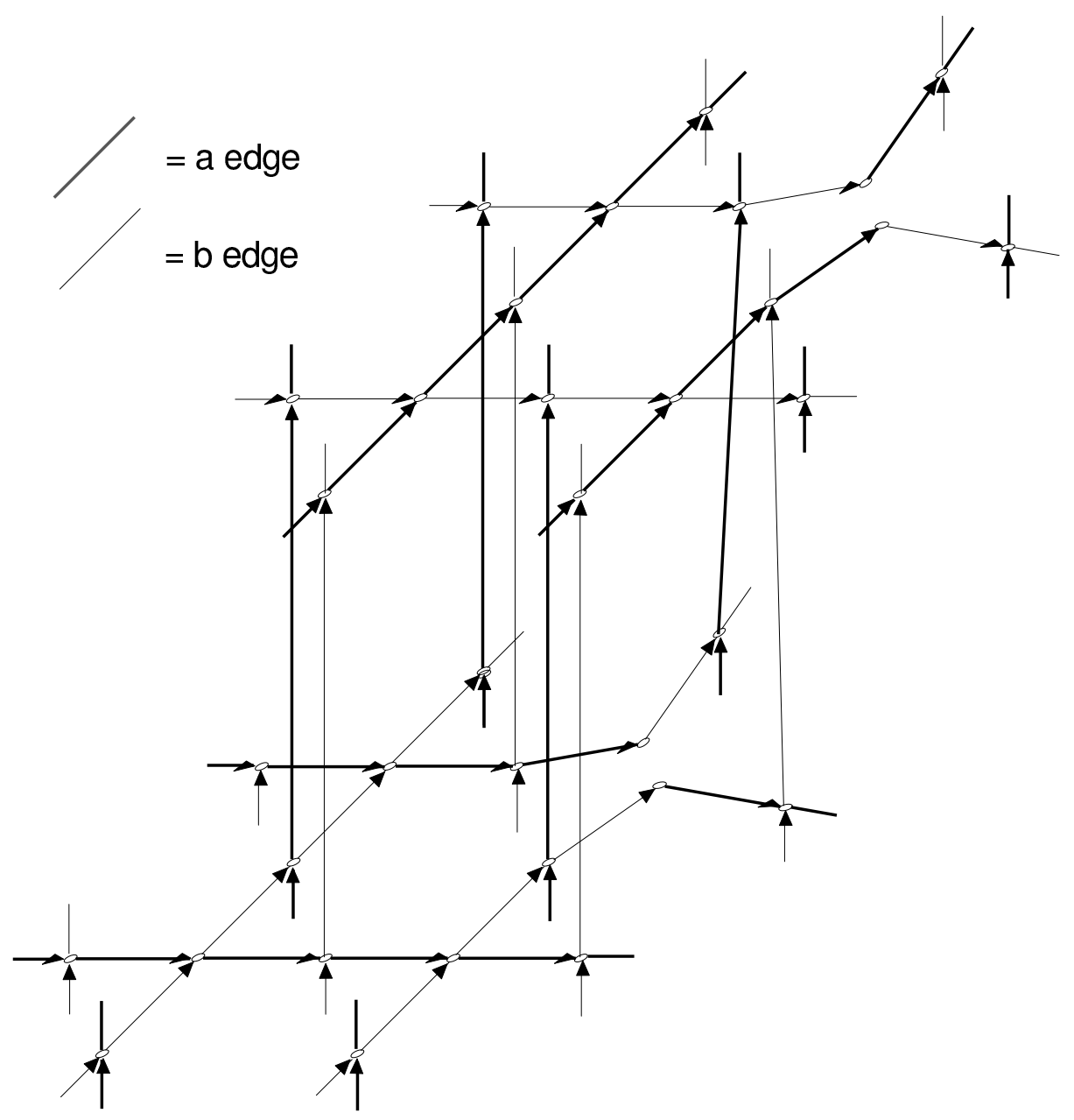

Figure 2. The Cayley graph of $B_{3}$.

Theorem 3.7. The graph $\Delta$ is the Cayley graph for $B_{3}$ with respect to the generating set $S=\{a, b\}^{ \pm 1}$.

Proof. By Proposition 3.6. vertices of $\Delta$ are in bijective correspondence with elements of $C F$, and elements of $R G$ are in bijective correspondence with vertices of the Cayley graph of $B_{3}$. Thus, to construct a bijection from $V \Delta$ to $V C_{B_{3}}$, we will define a bijection between $C F$ and $R G$.

We define a map $\Phi_{1}: C F \mapsto R G$ as the result of the following rewriting algorithm. Consider $w \in C F$, where $w=w^{\prime}(a b a)^{k}$, with $w^{\prime}$ almost even and $k \in \mathbb{Z}$.

First, this algorithm deals with every occurrence of $A B$ or $B A$ in $w^{\prime}$. These occurrences are non-overlapping since $w^{\prime}$ is almost even. For each occurrence of $A B$ in $w^{\prime}$, insert $b B$ beforehand, creating a power of $A B A$ : $A B \cong(b B)(A B)=b(B A B) \equiv b(A B A)$. Similarly, for every occurrence of 
$B A$ insert $a A$ beforehand, again creating a power of $A B A$. Then, pull all created occurrences of $A B A$ to the right end of $w$ using the Garside property. The result of the algorithm thus far is a word of the form $w^{\prime \prime}(a b a)^{k^{\prime}}$, where $w^{\prime \prime}$ contains no occurrences of $A B$ or $B A$; in other words, all negative syllables are isolated in $w^{\prime \prime}$. Note if $s^{c}(s \in\{a, b\}, c>0)$ is a syllable in $w^{\prime \prime}$ with $c=1$, then $s^{c}$ is the result of one of the insertions just described, and thus $s^{c}$ is between two negative syllables. For instance, $A A B B B B A A \mapsto$ $A(b B) A B B B(a A) B A A \mapsto A b A^{2} b A(A B A)^{2}$.

The second part of the algorithm deals with any remaining negative syllables in $w^{\prime \prime}$ as follows. For every occurrence of $A$, insert the trivial words $b B$ and $B b$ before and after the $A$, respectively, thus creating an occurrence of $B A B \equiv A B A$. Similarly, for every occurrence of $B$ insert $a A$ beforehand and $A a$ after. Finally, pull all powers of $A B A$ to the end of $w^{\prime \prime}$ using the Garside property. For instance, consider $w^{\prime \prime}=A^{c}$ for $c$ odd. We have $w^{\prime \prime}=A^{c} \equiv(b B A B b)(b B A B b) \ldots(b B A B b)$, where there are $c$ occurrences of $(b B A B b)$. Pulling each power of $B A B \equiv A B A$ to the end, we get $b a^{2} b^{2} \ldots b^{2} a(A B A)^{k}$. Note that for each negative syllable, this process creates occurrences of $A B A, a^{2}$, and $b^{2}$, and adds 1 to the power of adjacent syllables. Increasing the power of adjacent syllables eliminates the occurrences of syllables $s^{c}$ with $c=1$ in $w^{\prime \prime}$ created by the first part of the algorithm.

So, $w$ is equal in $B_{3}$ to the resultant word of the form $\tilde{w}:=w^{\prime \prime \prime}(a b a)^{c^{\prime \prime}}$, where $w^{\prime \prime \prime}$ has only positive syllables of exponent strictly greater than 1 (except perhaps the first or last syllables). Thus, $\tilde{w} \in R G$, and we set $\Phi_{1}(w)=\tilde{w}$.

We now define the map $\Phi_{2}: R G \mapsto C F$ by the following rewriting algorithm. Consider $\tilde{w} \in R G$, where $\tilde{w}=w^{\prime}(a b a)^{c}=a^{k_{1}} b^{k_{2}} \ldots a^{k_{n-1}} b^{k_{n}}(a b a)^{c}$ with $j \in \mathbb{Z}, k_{i}>1$ for $1<i<n$, and $k_{1}, k_{n} \geq 0$. If $k_{i}$ is even for each $1 \leq i<n$, then $\tilde{w}$ is already in $C F$, and we set $\Phi_{2}(\tilde{w})=\tilde{w}$. Otherwise, we proceed as follows.

Let $j=j_{w}$ be the least $i$ such that $k_{i}$ is odd in $w^{\prime}$. Without loss of generality assume the $j$ th syllable is $a^{k_{j}}$. If $k_{j+1}$ is even, then:

$$
\begin{aligned}
w & \equiv \ldots a^{k_{j}} b^{k_{j+1}} \ldots \\
& \equiv \ldots a^{k_{j}-1}(B B b b) a b b^{k_{j+1}-1} \ldots \\
& \equiv \ldots a^{k_{j}-1} B^{2} b a^{k_{j+1}-1}(b a b) \ldots \\
& \equiv \ldots a^{k_{j}-1} B^{2} A^{2} a a^{k_{j+1}-2}(b a b)^{2} \ldots \\
& \equiv \ldots a^{k_{j}-1} B^{2} A^{2} B^{2} b b^{k_{j+1}-3}(b a b)^{3} \ldots \\
& \equiv \ldots \\
& \equiv \ldots a^{k_{j}-1} B^{2} A^{2} B^{2} \ldots A^{2} B(a b a)^{k_{j+1}} \ldots
\end{aligned}
$$


where there are $k_{j+1}-2$ negative squared syllables. If $k_{j+1}$ is odd, then the result of the same process is $\ldots a^{k_{j}-1} B^{2} A^{2} B^{2} \ldots B^{2} A(a b a)^{k_{j+1}} \ldots$ Let $w^{\prime \prime}$ denote this result with the powers of $a b a$ pulled to the right end, using the Garside property. Then $\tilde{w} \equiv w^{\prime \prime}$. If $w^{\prime \prime}$ has $n^{\prime}$ syllables followed by $(a b a)^{c+k_{j+1}}$, then note $n-j_{w^{\prime}}>n^{\prime}-j_{w^{\prime \prime}}$. Thus this process will terminate upon iteration.

Continue to apply this algorithm to each resultant word until no odd syllables are left (except possibly the last one). The result of this process is a word of the form $w(a b a)^{c}$ where $w$ is almost even and $k \in \mathbb{Z}$, as desired. Set $\Phi_{2}(\tilde{w})=w(a b a)^{k}$.

Lemma 3.8. The functions $\Phi_{1}: C F \rightarrow R G$ and $\Phi_{2}: R G \rightarrow C F$ are bijections, and $\Phi_{2}=\Phi_{1}^{-1}$.

Proof. We have already defined maps $\Phi_{1}: C F \mapsto R G$ and $\Phi_{2}: R G \mapsto C F$. We need to show that $\Phi_{1} \circ \Phi_{2}(\tilde{w})=\tilde{w}$ for every $\tilde{w} \in R G$ and $\Phi_{2} \circ \Phi_{1}(w)=w$ for every $w \in C F$. Both $\Phi_{1}$ and $\Phi_{2}$ only use the relations of $B_{3}$; therefore, for any $\tilde{w} \in R G, \Phi_{1} \circ \Phi_{2}(\tilde{w}) \equiv \tilde{w}$. Since $R G$ is a set of canonical normal forms for $B_{3}$, this implies $\Phi_{1} \circ \Phi_{2}(\tilde{w})=\tilde{w}$. Thus it suffices to show $\Phi_{2} \circ \Phi_{1}(w)=w$ for every $w \in C F$.

Consider $\Phi_{2} \circ \Phi_{1}(w)$ for some non-trivial $w=w^{\prime}(a b a)^{k} \in C F$. Then $w$ is of the form $w_{1}^{+} w_{1}^{-} w_{2}^{+} w_{2}^{-} \ldots w_{m}^{+} w_{m}^{-}$for some $m \geq 0$, where each $w_{k}^{+}$(respectively, $w_{k}^{-}$) is a word with only positive (respectively, negative) syllables. We prove $\Phi_{2} \circ \Phi_{1}(w)=w$ by induction on $m$. Note if $m=0$ or $m=1$ and $w_{1}^{-}=1$ there is nothing to prove.

Assume that for $m=1, \ldots, n-1$ we have $\Phi_{2} \circ \Phi_{1}(w)=w$. For $m=n$, we have $w=w_{1}^{+} w_{1}^{-} w_{1}$ where $w_{1} \in C F$ and $\Phi_{2} \circ \Phi_{1}\left(w_{1}\right)=w_{1}$. Note

$$
\Phi_{2} \circ \Phi_{1}(w)=\Phi_{2}\left(w_{1}^{+} \Phi_{1}\left(w_{1}^{-} w_{1}\right)\right)=w_{1}^{+}\left[\Phi_{2} \circ \Phi_{1}\right]\left(w_{1}^{-} w_{1}\right),
$$

and $\Phi_{1}\left(w_{1}^{-} w_{1}\right)=\Phi_{1}\left(w_{1}^{-}\right) \Phi_{1}\left(w_{2}\right)$, where $w_{2}=w_{1}$ or $w_{2} \equiv \lambda\left(w_{1}\right)$. Thus, since $\Phi_{2}$ scans from left to right, if $\Phi_{2} \circ \Phi_{1}\left(w_{1}^{-}\right)=w_{1}^{-}$, then $\Phi_{2} \circ \Phi_{1}\left(w_{2}\right)=$ $\Phi_{2} \circ \Phi_{1}\left(w_{1}\right)=w_{1}$, and $\Phi_{2} \circ \Phi_{1}(w)=w$. So it suffices to show that $\Phi_{2} \circ$ $\Phi_{1}\left(w_{1}^{-}\right)=w_{1}^{-}$.

We will prove $\Phi_{2} \circ \Phi_{1}\left(w_{1}^{-}\right)=w_{1}^{-}$by induction on the number of negative syllables in $w_{1}^{-}$. Consider the number of (negative) syllables of $w_{1}^{-}$. Assume without loss of generality that the first syllable of $w_{1}^{-}$is $A^{2 k_{1}}, k_{1}>0$. If $w_{1}^{-}$ has only one syllable, then $\Phi_{1}\left(w_{1}^{-}\right)=b a^{2} b^{2} a^{2} \ldots a^{2} b(A B A)^{2 k_{1}}$, where there are $k_{1}$ occurrences of $a^{2}$, and: 


$$
\begin{aligned}
\Phi_{2}\left(\Phi_{1}\left(w_{1}^{-}\right)\right) & =\Phi_{2}\left(b a^{2} b^{2} a^{2} \ldots a^{2} b(A B A)^{2 k_{1}}\right) \\
& =A^{2} \Phi_{2}\left(a b a^{2} b^{2} a^{2} \ldots b^{2} a(A B A)^{2 k_{1}-1}\right) \\
& =A^{2} \Phi_{2}\left(B^{2} b b^{2} a^{2} b^{2} \ldots a^{2} b(A B A)^{2 k_{1}-2}\right) \\
& =A^{2} \Phi_{2}\left(b a^{2} b^{2} \ldots a^{2} b(A B A)^{2 k_{1}-2}\right) \\
& =\ldots \\
& =A^{2 k_{1}}
\end{aligned}
$$

as required.

If $w_{1}^{-}$has two negative syllables, it is of the form $A^{2 k_{1}} B^{2 k_{2}}$. Then the first part of the algorithm defining $\Phi_{1}$ eliminates the occurrence of $A B$, yielding $A^{2 k_{1}} b A^{2 k_{2}}(A B A)$. Then, $\Phi_{1}\left(w_{1}^{-}\right)=b a^{2} b^{2} \ldots b^{2} a^{3} b^{2} \ldots a^{2} b(A B A)^{2 k_{1}+2 k_{2}-1}$, where there are $k_{1}-1$ occurrences of $a^{2} b^{2}$ before the $a^{3}$ and $k_{2}-1$ occurrences of $b^{2} a^{2}$ after. Thus:

$$
\begin{aligned}
\Phi_{2}\left(\Phi_{1}\left(w_{1}^{-}\right)\right) & =\Phi_{2}\left(b a^{2} b^{2} a^{2} \ldots b^{2} a^{3} b^{2} a^{2} \ldots a^{2} b(A B A)^{2 k_{1}+2 k_{2}-1}\right) \\
& =A^{2} \Phi_{2}\left(b a^{2} \ldots b^{2} a^{3} b^{2} a^{2} \ldots a^{2} b(A B A)^{2 k_{1}+2 k_{2}-1-1}\right) \\
& =\ldots \\
& =A^{2 k_{1}-2} \Phi_{2}\left(b a^{3} b^{2} \ldots a^{2} b(A B A)^{2 k_{2}-1-\left(2 k_{1}-2\right)}\right) \\
& =A^{2 k_{1}-2} A^{2} \Phi_{2}\left(a(a b a) a a b^{2} \ldots a^{2} b(A B A)^{2 k_{2}+1}\right) \\
& =A^{2 k_{1}} \Phi_{2}\left(a b^{2} a^{2} \ldots b^{2} a(A B A)^{2 k_{2}}\right) \\
& =A^{2 k_{1}} B^{2 k_{2}}
\end{aligned}
$$

again as required.

If there are more than two negative syllables in $w_{1}^{-}$, then $w_{1}^{-}$is of the form $A^{2 k_{1}} B^{2 k_{2}} A^{2 k_{3}} \ldots$ Then, $\Phi_{1}\left(w_{1}^{-}\right)=b a^{2} b^{2} \ldots b^{2} a^{3} b^{2} a^{2} \ldots b^{2} a^{3} b^{2} a^{2} \ldots$, with $k_{j}-1$ occurrences of $b^{2}$ between the $(j-1)$ th and the $j$ th occurrence of $a^{3}$. When $\Phi_{2}$ is applied to this result, scanning from left to right we get

$$
\begin{aligned}
\Phi_{2}\left(\Phi_{1}\left(w_{1}^{-}\right)\right) & =\Phi_{2}\left(\Phi_{1}\left(A^{2 k_{1}} B^{2 k_{2}} A^{2 k_{3}} \ldots\right)\right) \\
& =\Phi_{2}\left(b a^{2} b^{2} \ldots b^{2} a^{3} b^{2} a^{2} \ldots b^{2} a^{3} b^{2} a^{2} \ldots\right) \\
& =A^{2 k_{1}} \Phi_{2}\left(a b^{2} a^{2} \ldots b^{2} a^{3} b^{2} a^{2} \ldots\right) \\
& =A^{2 k_{1}} \Phi_{2}\left(\Phi_{1}\left(B^{2 k_{2}} A^{2 k_{3}} \ldots\right)\right)
\end{aligned}
$$


and we may induct on the number of negative syllables of $w_{1}^{-}$. Thus $\Phi_{2}\left(\Phi_{1}\left(w_{1}^{-}\right)\right)$preserves $w_{1}^{-}$, and Lemma 3.8 is proved.

We now return to the proof of Theorem 3.7. It is left to prove there is a bijection between edges which preserves adjacency, i.e. that for $w \in C F$, if $\Phi_{1}(w)=u \in R G$, then $w a$ is mapped to $u a$, and $w b$ is mapped to $u b$. We claim that indeed $\Phi_{1}$ and $\Phi_{2}$ induce a graph isomorphism between $\Delta$ and $C_{B_{3}}$.

Let $w \in C F$ be mapped to $\Phi_{1}(w)=u \in R G$. For a given $x \in\{a, b\}^{ \pm 1}$, let $w_{1} \in C F$ and $u_{1} \in R G$ be such that $w_{1} \equiv w x$ and $u_{1} \equiv u x$. We want $\Phi_{1}\left(w_{1}\right)=u_{1}$ if and only if $\Phi_{1}(w) x \equiv \Phi_{1}\left(w_{1}\right)$.

There are many cases to deal with for this argument. We deal with two; the rest may be argued similarly. Assume without loss of generality that $x=a$ and $w$ is of the form $w=\ldots b^{k_{n-1}} a^{k_{n}}(a b a)^{i}$, with $k_{i}$ even for $1 \leq i<n$. Look at $\Phi_{1}\left(w_{1}\right)$. Assume $k_{n}<0, k_{n}$ even, $k_{n-1}>0$, and $j_{u}$ even. Since $k_{n}<0, u=\ldots a^{2} b(a b a)^{j}$. Then:

- If $j_{w}$ is even, then, in $B_{3}, w a \equiv \ldots a^{k_{n}}(a b a)^{j_{w}} a \equiv \ldots a^{k_{n}+1}(a b a)^{j_{w}} \in$ $C F$, so $w_{1}$ is of the form $\ldots a^{k_{n}+1}(a b a)^{j_{w}}$. But

$$
\Phi_{1}\left(w_{1}\right) \equiv \ldots a(a b a)^{j_{u}+1} \equiv \ldots a^{2} b a(a b a)^{j_{u}} \equiv a^{2} b(a b a)^{j_{u}} a \equiv u a=\Phi_{1}(w) a
$$

by definition, and so $u_{1}=\Phi_{1}\left(w_{1}\right)$ as required.

- If $j_{w}$ is odd, then, in $B_{3}, w a \equiv \ldots a^{k_{n}}(a b a)^{j_{w}} a \equiv \ldots a^{k_{n}} b(a b a)^{j_{w}} \in$ $C F$ since $k_{n}$ is even. Thus $w_{1}$ is of the form ... $a^{k_{n}} b(a b a)^{j_{w}}$. Again,

$$
\Phi_{1}\left(w_{1}\right) \equiv \ldots a^{2} b^{2}(a b a)^{j_{u}} \equiv \ldots a^{2} b(a b a)^{j_{u}} a \equiv u a=\Phi_{1}(w) a
$$

and so $u_{1}=\Phi_{1}\left(w_{1}\right)$ as required.

In every case we see $\Phi_{1}$ preserves vertex adjacency, making a bijection between the two graphs which preserves adjacency. Thus, $\Delta \cong C_{B_{3}}$, and the proof of Theorem 3.7 is complete.

\section{Characterizing Geodesics of $B_{3}$}

4.1. Some Definitions. Looking at the Cayley graph $\Delta$ of $B_{3}$, one might think that a word $w=w^{\prime}(a b a)^{k} \in C F$ (where $w^{\prime}$ is almost even and $k \in \mathbb{Z}$ ) is geodesic. However, this is not necessarily the case. For instance, the word $a^{2} b^{2} A^{2} B^{2} \in C F$ is equal in $B_{3}$ to the shorter word $a B a B a B$.

It is sometimes shorter in $\Delta$ to move from the translate $T^{\prime} \times\{k\}$ of $T^{\prime}$ to a nearby translate $T^{\prime} \times\{k \pm 1\}$ and then back. Algebraically, we see that if $w$ is of the form $w=\ldots(a b) b^{k_{1}} a^{k_{2}} \ldots a^{k_{n}}(A B) \ldots$, then, 


$$
\begin{aligned}
w & =\ldots(a b) b^{k_{1}} a^{k_{2}} \ldots a^{k_{n}}(A B) \ldots \\
& \equiv \ldots B(b a b) b^{k_{1}} a^{k_{2}} \ldots a^{k_{n}}(A B A) a \ldots \\
& \equiv \ldots B a^{k_{1}} b^{k_{2}} \ldots b^{k_{n}} a \ldots
\end{aligned}
$$

by the Garside property. This gives a shorter representation, so if $w$ contains both $a b$ and $A B$, then $w$ is not geodesic. In fact, if $w$ contains both one of $\{a b, b a\}$ and one of $\{A B, B A\}$ as subwords, then a similar argument proves $w$ is not geodesic.

Definition $4.1\left(*^{+}\right.$and $\left.*^{-}\right)$. Let $*^{+}:=\{a b, b a\}$ and $*^{-}:=\{A B, B A\}$.

Thus a geodesic may not have as subwords elements of both $*^{+}$and of $*^{-}$. Indeed, also by the Garside property, a geodesic may not have as subwords both $(a b a)$ and either $A$ or $B$, and it may not have as subwords both $(A B A)$ and either $a$ or $b$. This motivates the following:

Definition 4.2 (The ${ }^{*}$ condition). An element $w \in S^{*}$ is said to satisfy the * condition if $w$ does not contain as subwords elements of both $*^{+}$and $*^{-}$.

Definition 4.3 (The ${ }^{* *}$ condition). An element $w \in S^{*}$ is said to satisfy the ${ }^{* *}$ condition if:

- $w$ does not contain as subwords both $(a b a)$ and either $A$ or $B$, and

- $w$ does not contain as subwords both $(A B A)$ and either $a$ or $b$.

Definition $4.4(\Omega)$. We define $\Omega$ to be the set of all $w \in S^{*}$ such that $w$ is freely reduced and satisfies both the ${ }^{*}$ condition and the ${ }^{* *}$ condition.

The following is now obvious:

Theorem 4.5. We have that $L_{B_{3}} \subseteq \Omega$.

The ${ }^{*}$ and ${ }^{* *}$ conditions thus severely limit the set of possible geodesics. They are necessary conditions on a geodesic; we will eventually see that they are also sufficient.

Recall that in Section 2 we discussed the short-lex ordering on $S^{*}$. Fix the lexicographical ordering $a<A<b<B$ on $S=\{a, b\}^{ \pm 1}$. Let $S L$ denote the set of short-lex normal forms for $B_{3}$.

Lemma 4.6. A word $w$ is in $S L$ if and only if it is in one of the following forms:

- $\left(a^{i}\right)\left(b^{j_{1}} A^{j_{2}} b^{j_{3}} \ldots A^{j_{m-1}} b^{j_{m}}\right)\left(a^{k_{1}} b^{k_{2}} a^{k_{3}} \ldots a^{k_{n}} b^{k}\right)$,

- $\left(a^{i}\right)\left(b^{j_{1}} A^{j_{2}} b^{j_{3}} \ldots A^{j_{m-1}} b^{j_{m}}\right)\left(a^{k_{1}} b^{k_{2}} a^{k_{3}} \ldots b^{k_{n}} a^{k}\right)$,

- $\left(a^{i}\right)\left(B^{j_{1}} a^{j_{2}} B^{j_{3}} \ldots a^{j_{m-1}} B^{j_{m}}\right)\left(A^{k_{1}} B^{k_{2}} A^{k_{3}} \ldots A^{k_{n}} B^{k}\right)$, or

- $\left(a^{i}\right)\left(B^{j_{1}} a^{j_{2}} B^{j_{3}} \ldots a^{j_{m-1}} B^{j_{m}}\right)\left(A^{k_{1}} B^{k_{2}} A^{k_{3}} \ldots B^{k_{n}} A^{k}\right)$,

where $i \in \mathbb{Z}, m$ and $n$ are non-negative integers, $j_{l}>0$ for every $0 \leq l \leq m$, $k_{l}>1$ for every $0 \leq l \leq n$, and $k$ is either 0 or 1 . 
Proof. These are computed from the FSA for $S L$, which was computed by the program KBMAG.

Note that $S L$ is a subset of the set of geodesics of $B_{3}$.

4.2. Proving $\Omega=L_{B_{3}}$. Recall that the set of geodesic words of $B_{3}$ is $L_{B_{3}}$. With the definitions above, we may now restate Theorem 1.1 with simpler notation:

Theorem 4.7. We have that $\Omega=L_{B_{3}}$. In other words, a freely reduced word $w \in S^{*}$ is geodesic in $B_{3}$ if and only if $w$ satisfies the ${ }^{*}$ and ${ }^{* *}$ conditions.

Proof. By Theorem 4.5, we have that $L_{B_{3}} \subseteq \Omega$. It remains to prove that $\Omega \subseteq L_{B_{3}}$. We do so by showing that a word $w$ in $\Omega$ is equal in $B_{3}$ to a word $w^{\prime}$ in $S L$ obtained from $w$ by a length-preserving map. We present an algorithm for rewriting $w$ to $w^{\prime}$ in two steps. The first step takes a word $w \in \Omega$ into a temporary form (increasing the length by a certain amount), and the second step maps words in the temporary form to $S L$ (decreasing the length by the same amount).

Let $T F$ denote the set of all words of the form $\left(x^{k_{1}} Y^{k_{2}} \ldots Y^{k_{n-1}} x^{k_{n}}\right)(a b a)^{j}$, with $k_{n} \geq 0, k_{i} \geq 1$ for $1 \leq i<n$, and for some $(x, y) \in\{(a, b),(b, a)\}^{ \pm 1}$. We will say that a word in $T F$ is in temporary form.

Define $\Psi_{1}: S^{*} \rightarrow T F$ by the following algorithm. For a word $w \in S^{*}$, first freely reduce $w$. Then, pull all subwords of the form $a b a, b a b, A B A$, and $B A B$ to the right end using the Garside property, so $w \equiv w^{\prime}(a b a)^{j^{\prime}}$, and freely reduce $w^{\prime}$. Then, look at $w^{\prime}$ in terms of its syllables. Working from left to right, if there are ever two syllables in a row of the form $x^{k_{i}} y^{k_{i+1}}$ with $(x, y) \in\{(a, b),(b, a)\}^{ \pm 1}$, then since

$$
\begin{aligned}
x^{k_{i}} y^{k_{i+1}} & =x^{k_{i}-1} x y y^{k_{i+1}-1} \\
& \equiv x^{k_{i}-1} Y(y x y) y^{k_{i+1}-1} \\
& \equiv x^{k_{i}-1} Y x^{k_{i+1}-1}(y x y),
\end{aligned}
$$

we may replace $x^{k_{i}} y^{k_{i+1}}$ with $x^{k_{i}-1} Y x^{k_{i+1}-1}(y x y)$ and pull the power of $(a b a)$ out of $w^{\prime}$ to the end of $w$. Then repeat this replacement for every appropriate pair of syllables, working to the end of $w^{\prime}$. The resulting word is $\Psi_{1}(w)$.

There are a few things to note about $\Psi_{1}$ concerning the length in $B_{3}$ of $\Psi_{1}(w)$. The initial process of moving occurrences of $(x y x)$ to the end of the word does not change the length of a freely reduced word $w$, unless there are both positive and negative powers of $a b a$ in the original word. For $w \in \Omega$, however, this does not happen by the ${ }^{* *}$ condition. For the remainder of the algorithm, each described replacement increases by two the length of the resultant word, unless cancellation occurs. Cancellation may occur in two locations. If the power of $a b a$ pulled to the end has opposite sign of another power of $a b a$ also pulled to the end, then cancellation occurs. However, this only happens when initially $w$ had as subwords elements of both $*^{+}$and $*^{-}$. 
This contradicts the ${ }^{*}$ condition, so for $w \in \Omega$, cancellation does not occur at the end of the word. Cancellation could also occur if $k_{i}$ or $k_{i+1}$ equals 1 . If $k_{i}=1$ and the $i$ th syllable is $x$, and if the $(i-1)$ th syllable of $w$ exists and is $y^{k_{i-1}}$ with $k_{i-1}>0$, then $Y$ will cancel with the $(i-1)$ th syllable. Similarly, if $k_{i+1}=1$ and if the $(i+2)$ th syllable exists and is a positive power of $x$, cancellation will again occur. However, in each case $w^{\prime}$ would have to have a subword of the form $y x y$ or $x y x$, respectively, and by the first part of the algorithm, this does not happen. Thus, for $w \in \Omega$, the length of $\Psi_{1}(w)$ is exactly the length of $w$ plus twice the number of occurrences of subwords of the form $a b, b a, A B$, and $B A$. Let $\lambda$ denote the number of such subwords.

We define $\Psi_{2}: T F \rightarrow S L$ as the result of the following rewriting algorithm. Consider a word $w^{\prime} \in T F$. Then $w^{\prime}$ is of the form

$$
\left(x^{k_{1}} Y^{k_{2}} \ldots Y^{k_{n-1}} x^{k_{n}}\right)(a b a)^{j}
$$

for some $(x, y) \in\{(a, b),(b, a)\}^{ \pm 1}$. Note that $x^{k_{1}} Y^{k_{2}} \ldots Y^{k_{n-1}} x^{k_{n}} \in S L$, so if $j=0$, define $\Psi_{2}\left(w^{\prime}\right)=w^{\prime}$. For $j \neq 0$, without loss of generality assume $j$ is positive and assume $x=a$ (for the other seven combinations, similar definitions of $\Psi_{2}$ may be made, but are not elaborated upon here). Then $w^{\prime}$ is of the form $\left(a^{k_{1}} B^{k_{2}} \ldots B^{k_{n-1}} a^{k_{n}}\right)(a b a)^{j}$. It is still true that $a^{k_{1}} B^{k_{2}} \ldots B^{k_{n-1}} a^{k_{n}} \in S L$; thus, we need to deal with $(a b a)^{j}$. First, move one of the powers of $(a b a)$ before the first negative syllable and freely reduce (if no such syllable exists, skip this step):

$$
\begin{aligned}
w^{\prime} & =\left(a^{k_{1}} B^{k_{2}} a^{k_{3}} \ldots a^{k_{n-2}} B^{k_{n-1}} a^{k_{n}}\right)(a b a)^{j} \\
& \equiv\left(a^{k_{1}}(a b a) A^{k_{2}} b^{k_{3}} \ldots b^{k_{n-2}} A^{k_{n-1}} b^{k_{n}}\right)(a b a)^{j-1} \\
& \equiv\left(a^{k_{1}+1} b A^{k_{2}-1} b^{k_{3}} \ldots b^{k_{n-2}} A^{k_{n-1}} b^{k_{n}}\right)(a b a)^{j-1} \\
& \equiv\left(a^{k_{1}+1}\right)\left(b A^{k_{2}-1} b^{k_{3}} \ldots b^{k_{n-2}} A^{k_{n-1}} b^{k_{n}}\right)(a b a)^{j-1} .
\end{aligned}
$$

Again, $\left(a^{k_{1}+1}\right)\left(b A^{k_{2}-1} b^{k_{3}} \ldots b^{k_{n-2}} A^{k_{n-1}} b^{k_{n}}\right) \in S L$, and the length of the word is decreased by two.

If $j>1$, then the remaining powers of $(a b a)$ will be eliminated as follows. While there are still powers of $(a b a)$ left, if there are any negative syllables remaining, take an $(a b a)$ and place it after the last negative syllable using the Garside property and then freely reduce. For instance, for $w=\left(a^{2} B^{2} a B^{2}\right)(a b a)^{2}$, initially we make the word $\left(a^{2}(a b a) A^{2} b A^{2}\right)(a b a)=$ $\left(a^{3}\right)\left(b A b A^{2}\right)(a b a)$, and then $\left(a^{3}\right)\left(b A b A^{2}(a b a)\right)=\left(a^{3}\right)(b A b A b)(a)$ (which is in short-lex form). We may continue to reduce the exponent on $(a b a)$ in this process, each time decreasing the length of the word by two, until either there are no more $(a b a) \mathrm{s}$, or there are no more negative syllables remaining. Let $\alpha$ denote the number of occurrences of $A$ and $B$ originally in $w$. Then thus far we have decreased the length of the word by $\min \{\alpha, j\}$.

If $j>\alpha$, to eliminate the remaining powers of $(a b a)$, insert them one by one after the first syllable. In short-lex normal form, only the second 
syllable may be raised to the first power (see Definition 4.6). If we begin with a word in $S L$, though, this algorithm preserves that property, and the result is in $S L$. In total, then, $\Psi_{2}$ shortens a word $w^{\prime} \in T F$ by $2 * \min \{\alpha, j\}$.

Now consider the map $\Psi:=\Psi_{2} \circ \Psi_{1}$. Let $w_{1}:=\Psi_{1}(w)$ and $w_{2}:=$ $\Psi_{2}\left(w_{1}\right)=\Psi(w)$. When restricted to $w \in \Omega$, as already noted for $w_{1}, \Psi_{1}$ first pulls out all powers of ( $a b a)$ (preserving the length), and then pulls out all subwords of the form $a b, b a, A B$, or $B A$, each time increasing the length of the word by two. For a word in $\Omega$, if there exist any subwords of the form $a b a$ or $b a b$, then by the ${ }^{* *}$ condition there are no negative powers in $w$, and $j \geq \alpha=\lambda$, so

$$
\left|w_{2}\right|=\left|w_{1}\right|-2 * \min (\alpha, j)=(|w|+2 * \lambda)-2 * \alpha=|w|
$$

and in this case $\Psi$ preserves the length of $w$.

If $w$ does not have any subwords of the form $a b a$ or $b a b$, then either $w$ is already in $S L$ (as shown above) or $w$ contains subwords from exactly one of $*^{+}$or $*^{-}$. Without loss of generality, assume $w$ contains elements from only $*^{+}$. Then $j=\lambda$ (recall $j$ the exponent of $(a b a)$ in $w_{1}=\Psi(w)$ ). Furthermore, for every occurrence of an element of $*^{+}$in $w, \Psi_{1}$ inserts exactly one negative letter which does not cancel with its neighbors. Thus we have $\alpha \geq j$ (recall $\alpha$ is the number of negative elements in $w_{1}$ ), and $\left|w_{2}\right|=\left|w_{1}\right|-2 * \min \{\alpha, j\}=(|w|+2 * \lambda)-2 * j=|w|$, as desired.

Thus, for any $w \in \Omega,|\Psi(w)|=|w|$, and $\Psi$ restricted to $\Omega$ is a lengthpreserving map, and we have proven Theorem 1.1 .

4.3. Translation lengths in $B_{3}$. For a general group $G$ with generating set $S$, we define the length $|g|_{S}$ of an element $g \in G$ with respect to $S$ :

$$
|g|_{S}:=\inf _{w \in S^{*}, \bar{w}=g}\{|w|\} .
$$

The translation length of an element $g \in G$ is defined to be:

$$
\tau_{S}(g):=\limsup _{n \rightarrow \infty} \frac{\left|g^{n}\right|_{S}}{n} .
$$

It is well known that the limit here always exists, by subadditivity. It is straightforward to check that that $\tau_{S}(g)$ depends only on the conjugacy class $C C(g)$ of $g$, where $C C(g):=\left\{h^{-1} g h \mid h \in G\right\}$. For more information on translation numbers, see for instance [10, [5], or [12].

It is known that for any word-hyperbolic group $G$, for any finite generating set $S$ of $G$ and any $g \in G, \tau_{S}(g)$ is a rational number [1] the denominator of $\tau_{S}(g)$ is bounded (stated by Gromov; proved in [18]). The previous results of this section now also allow us to prove:

Corollary 4.8. For $S=\{a, b\}^{ \pm 1}$ and any element $g \in B_{3}, \tau_{S}(g)$ is an integer. In particular, $\tau_{S}(g)=\left|g^{\prime}\right|_{S}$, where $g^{\prime}$ is a shortest element in $C C(g)$. 
Proof. Consider a general element $g \in B_{3}$. We may assume that $g$ is the smallest element in its conjugacy class. Then a word $w \in S^{*}$ representing $g$ is geodesic if and only if $w$ is cyclically reduced, any cyclic permutation of $w$ is geodesic, and $w$ satisfies the ${ }^{*}$ and ${ }^{*}$ conditions. For any $n \in \mathbb{N}$, if $w^{n}$ is not geodesic, then $w^{n}$ violates either the ${ }^{*}$ or the ${ }^{* *}$ condition. By the periodicity of $w^{n}$, there exists some subword $w^{\prime}$ of $w^{n}$ of length $|w|$ which violates either the ${ }^{*}$ or the ${ }^{* *}$ condition. But then $w^{\prime}$ is a cyclic permutation of $w$ which is not geodesic, contradicting the choice of $g$. Thus $w^{n}$ is a geodesic representative of $g^{n}$, and so $\left|g^{n}\right|_{S}=n|w|$ for each $n$, and $\tau_{S}(g)=|w|=|g|_{S}$.

\section{Computing the Growth Series}

5.1. The FSA for Geodesics of $B_{3}$. By Theorem 1.1 we know that a freely reduced word $w \in S^{*}$ is a geodesic in $B_{3}$ if and only if $w$ satisfies the $*$ and ${ }^{* *}$ conditions. Using this knowledge, constructing the FSA accepting geodesic words for $B_{3}$ is straightforward - the FSA simply needs to keep track of which choices have been made with respect to the ${ }^{*}$ and ${ }^{* *}$ condition. It is clear that checking whether a word satisfies the ${ }^{*}$ and ${ }^{* *}$ conditions requires keeping track of only a finite amount of information, so such an FSA exists. We have:

Theorem 5.1. The FSA in Figure 3 accepts a word $w \in S^{*}$ if and only if $w$ is a geodesic of $B_{3}$. Thus the set of geodesics $L_{B_{3}}$ of $B_{3}$ is regular and $\mathcal{G}_{\left(B_{3}, S\right)}$ is rational.

The different parts of Figure 3 represent which decisions have been made by the automaton when reading a word $w \in \Omega$ with respect to the ${ }^{*}$ and $* *$ condition. The decisions made are described below:

- Part I: $w$ has as a subword an element of $*^{+}$.

- Part II: $w$ has as a subword an element of $*^{-}$.

- Part III: $w$ has as subwords $a$ and $B$, but no elements of $*^{+}$or $*^{-}$.

- Part IV: $w$ has as subwords $b$ and $A$, but no elements of $*^{+}$or $*^{-}$.

- Part V: $w$ has as a subword either $a b a$ or $b a b$.

- Part VI: $w$ has as a subword either $A B A$ or $B A B$.

- Part VII: $w$ has as subwords an element of $*^{+}$and a negative syllable.

- Part VIII: $w$ has as subwords an element of $*^{-}$and a positive syllable.

5.2. The Geodesic Growth Series of $B_{3}$. Recall that by Proposition 2.1 we have

$$
\mathcal{G}_{\left(G_{3}, S\right)}(x)=v_{1}(I-M x)^{-1} v_{2}
$$

with $I, M, v_{1}$ and $v_{2}$ as in Proposition 2.1

When the adjacency matrix of the FSA for $B_{3}$ is plugged into this formula using Maple 4.5, we get: 


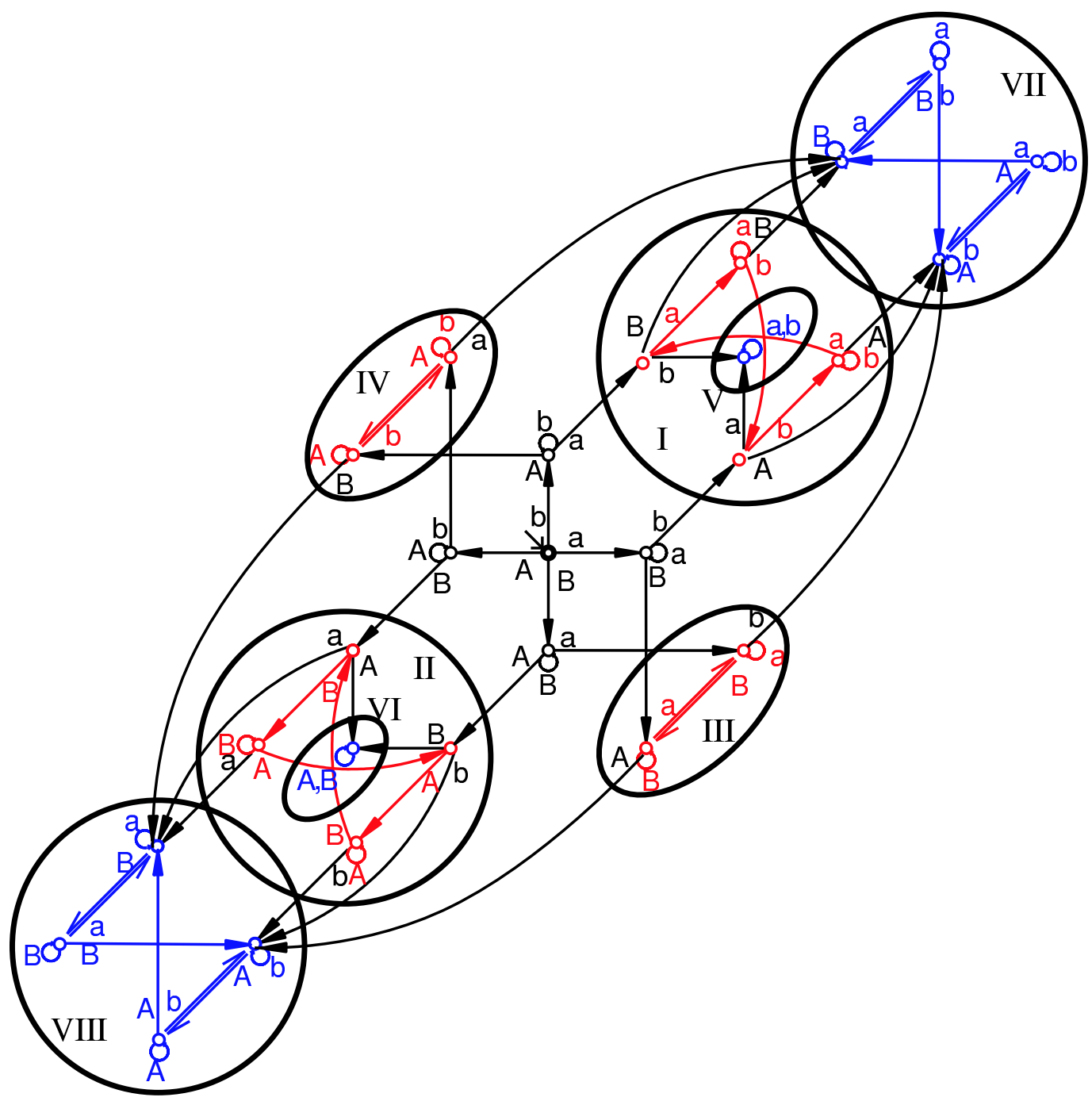

Figure 3 . The FSA which accepts geodesics of $B_{3}$, with its different components labelled. All states shown are accept states; the single fail state is not shown. There are 27 states.

Theorem 5.2. (c.f. Theorem 1.2) The Geodesic Growth Series of $B_{3}$ with respect to $S=\{a, b\}^{ \pm 1}$ is

$$
\mathcal{G}_{\left(B_{3}, S\right)}(x)=\frac{x^{4}+3 x^{3}+x+1}{\left(x^{2}+x-1\right)\left(x^{2}+2 x-1\right)} .
$$

For purposes of comparison, using the method proved in [2] and using the FSA which accepts the short-lex normal form of $B_{3}$ computed by the program KBMAG (see [8]), we have: 
Theorem 5.3. The Spherical Growth Series of $B_{3}$ is

$$
\mathcal{S}_{\left(B_{3}, S\right)}(x)=\frac{2 x^{4}+x^{3}-1}{\left(2 x^{3}+x^{2}-3 x+1\right)(x-1)} .
$$

\section{REFERENCES}

[1] G. Baumslag, S. M. Gersten, M. Shapiro, and H. Short. Automatic groups and amalgams. J. Pure Appl. Algebra, 76(3):229-316, 1991.

[2] Marcus Brazil. Monoid growth functions for braid groups. Int. J. of Algebra and Comp., 1(4):201-205, 1991.

[3] James W. Cannon. The combinatorial structure of cocompact discrete hyperbolic groups. Geom. Dedicata, 16(2):123-148, 1984.

[4] Ruth Charney and John Meier. The language of geodesics for Garside groups. Math. $Z$., to appear.

[5] Gregory R. Conner. Translation numbers of groups acting on quasiconvex spaces. In Computational and geometric aspects of modern algebra (Edinburgh, 1998), volume 275 of London Math. Soc. Lecture Note Ser., pages 28-38. Cambridge Univ. Press, Cambridge, 2000.

[6] John Crisp and Luis Paris. The solution to a conjecture of Tits on the subgroup generated by the squares of the generators of an Artin group. Invent. Math., 145(1):19-36, 2001.

[7] Carl Droms, Jacques Lewin, and Herman Servatius. The Tits conjecture and the five string braid group. In Topology and combinatorial group theory (Hanover, NH, 1986/1987; Enfield, NH, 1988), pages 48-51. Springer, Berlin, 1990.

[8] D. B. A. Epstein, D. F. Holt, and S. E. Rees. The use of Knuth-Bendix methods to solve the word problem in automatic groups. J. Symbolic Comput., 12(4-5):397-414, 1991. Computational group theory, Part 2.

[9] David B. A. Epstein, James W. Cannon, Derek F. Holt, Silvio V. F. Levy, Michael S. Paterson, and William P. Thurston. Word processing in groups. Jones and Bartlett Publishers, Boston, MA, 1992.

[10] S. M. Gersten and H. B. Short. Rational subgroups of biautomatic groups. Ann. of Math. (2), 134(1):125-158, 1991.

[11] Alex Griffing, Jon McCammond, Matthew Patlovany, and Jacob Talley. Private communication.

[12] Ilya Kapovich. Small cancellation groups and translation numbers. Trans. Amer. Math. Soc., 349(5):1851-1875, 1997.

[13] Joseph Loeffler, John Meier, and James Worthington. Graph products and Cannon pairs. Internat. J. Algebra Comput., 12(6):747-754, 2002.

[14] Walter D. Neumann and Michael Shapiro. Automatic structures, rational growth, and geometrically finite hyperbolic groups. Invent. Math., 120(2):259-287, 1995.

[15] Gen. A. Noskov. Growth of certain non-positively curved cube groups. European J. Combin., 21(5):659-666, 2000.

[16] Lucas Sabalka. Geodesics in the braid group on three strands and a related group. Undergraduate Thesis, University of Nebraska-Lincoln, 2002.

[17] Michael Stoll. Rational and transcendental growth series for the higher heisenberg groups. Invent. Math., 126(1):85-109, 1996.

[18] Eric L. Swenson. Hyperbolic elements in negatively curved groups. Geom. Dedicata, 55(2):199-210, 1995.

Deptartment of Mathematics, University of Illinois at Urbana-Champaign, Champaign, IL 61820

E-mail address: sabalka@math.uiuc.edu 